Please do not remove this page

RMIT

UNIVERSITY

\title{
Towards achieving strong coupling in three-dimensional-cavity with solid state spin resonance
}

Le Floch, Jean; Delhote, Nicolas; Aubourg, Michel; Madrangeas, Valérie; Cros, Dominique; Castelletto, Stefania; Tobar, Michael

https://researchrepository.rmit.edu.au/esploro/outputs/9921860278101341/filesAndLinks?institution=61RMIT_INST\&index=null

Le Floch, J., Delhote, N., Aubourg, M., Madrangeas, V., Cros, D., Castelletto, S., \& Tobar, M. (2016). Towards achieving strong coupling in three-dimensional-cavity with solid state spin resonance. Journal of Applied Physics, 119(15), 1-8. https://doi.org/10.1063/1.4946893

Document Version: Published Version

Published Version: https://doi.org/10.1063/1.4946893

Repository homepage: https://researchrepository.rmit.edu.au (c) 2016 Author(s).

Downloaded On 2023/04/26 23:07:52 +1000 
Thank you for downloading this document from the RMIT Research Repository.

The RMIT Research Repository is an open access database showcasing the research outputs of RMIT University researchers.

RMIT Research Repository: http://researchbank.rmit.edu.aul

\section{Citation:}

Le Floch, J, Delhote, N, Aubourg, M, Madrangeas, V, Cros, D, Castelletto, S and Tobar, M 2016, 'Towards achieving strong coupling in three-dimensional-cavity with solid state spin resonance', Journal of Applied Physics, vol. 119, no. 15, 153901, pp. 1-8.

See this record in the RMIT Research Repository at:

https://researchbank.rmit.edu.au/view/rmit:37434

Version: Published Version

Copyright Statement:

(C) 2016 Author(s).

\section{Link to Published Version:}

http://dx.doi.org/10.1063/1.4946893 


\section{Towards achieving strong coupling in three- dimensional-cavity with solid state spin resonance}

Cite as: J. Appl. Phys. 119, 153901 (2016); https://doi.org/10.1063/1.4946893

Submitted: 07 December 2015 . Accepted: 01 April 2016 . Published Online: 15 April 2016

J.-M. Le Floch (D), N. Delhote (D), M. Aubourg, V. Madrangeas, D. Cros, S. Castelletto (D), and M. E. Tobar
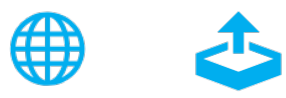

View Online

Export Citation

\section{ARTICLES YOU MAY BE INTERESTED IN}

Collective strong coupling with homogeneous Rabi frequencies using a 3D lumped element microwave resonator

Applied Physics Letters 109, 033508 (2016); https://doi.org/10.1063/1.4959095

Superstrong coupling of a microwave cavity to yttrium iron garnet magnons

Applied Physics Letters 108, 062402 (2016); https://doi.org/10.1063/1.4941730

Electron spin ensemble strongly coupled to a three-dimensional microwave cavity

Applied Physics Letters 98, 251108 (2011); https://doi.org/10.1063/1.3601930

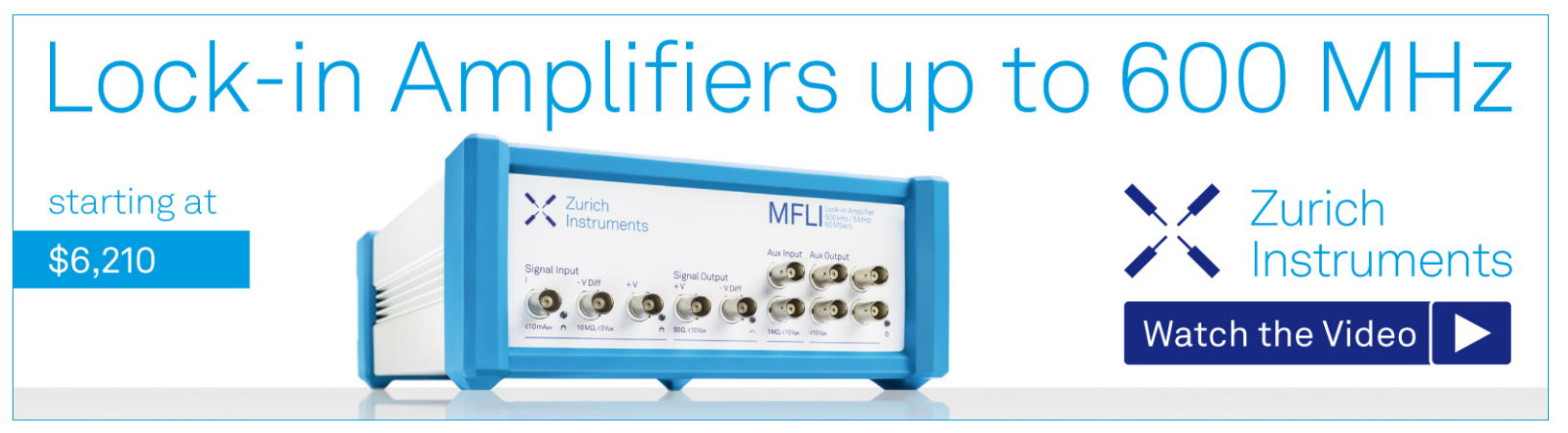




\title{
Towards achieving strong coupling in three-dimensional-cavity with solid state spin resonance
}

\author{
J.-M. Le Floch, ${ }^{1,2,3, a)}$ N. Delhote, ${ }^{4}$ M. Aubourg, ${ }^{4}$ V. Madrangeas, ${ }^{4}$ D. Cros,${ }^{4}$ S. Castelletto, ${ }^{5}$ \\ and M. E. Tobar ${ }^{2,3}$ \\ ${ }^{1}$ MOE Key Laboratory of Fundamental Physical Quantities Measurement, School of Physics, \\ Huazhong University of Science and Technology, Wuhan 430074, Hubei, China \\ ${ }^{2}$ School of Physics, The University of Western Australia, Crawley, Western Australia 6009, Australia \\ ${ }^{3}$ ARC Centre of Excellence for Engineered Quantum Systems, Crawley, Western Australia 6009, Australia \\ ${ }^{4}$ XLIM, UMR CNRS 7252, Université de Limoges, 123 av. A. Thomas, 87060 Limoges Cedex, France \\ ${ }^{5}$ School of Aerospace, Mechanical and Manufacturing Engineering, RMIT University, Melbourne, Australia
}

(Received 7 December 2015; accepted 1 April 2016; published online 15 April 2016)

\begin{abstract}
We investigate the microwave magnetic field confinement in several microwave three-dimensional (3D)-cavities, using a 3D finite-element analysis to determine the best design and achieve a strong coupling between microwave resonant cavity photons and solid state spins. Specifically, we design cavities for achieving strong coupling of electromagnetic modes with an ensemble of nitrogen vacancy (NV) defects in diamond. We report here a novel and practical cavity design with a magnetic filling factor of up to 4 times (2 times higher collective coupling) than previously achieved using one-dimensional superconducting cavities with a small mode volume. In addition, we show that by using a double-split resonator cavity, it is possible to achieve up to 200 times better cooperative factor than the currently demonstrated with NV in diamond. These designs open up further opportunities for studying strong and ultra-strong coupling effects on spins in solids using alternative systems with a wider range of design parameters. The strong coupling of paramagnetic spin defects with a photonic cavity is used in quantum computer architecture, to interface electrons spins with photons, facilitating their read-out and processing of quantum information. To achieve this, the combination of collective coupling of spins and cavity mode is more feasible and offers a promising method. This is a relevant milestone to develop advanced quantum technology and to test fundamental physics principles. Published by AIP Publishing.
\end{abstract}

[http://dx.doi.org/10.1063/1.4946893]

\section{INTRODUCTION}

In recent years, paramagnetic spin defects in semiconductors and their quantum control at room temperature make them among the most relevant candidates for future scalable quantum computing. ${ }^{1}$ For instance, spin defects in solid state hold promising applications spanning from ideal qubits ${ }^{2}$ to unique magnetic resonance imaging and temperature probes. ${ }^{3,4}$ One way to increase the coupling between systems is to use a large number of spins at once (collective coupling) coupled with either photonic or microwave cavities. The collective coupling, $g_{c}$, with the resonator mode can be enhanced by $g_{c}=g_{s} \sqrt{N}$, , 6 where $N$ is the number of identical two-level systems available (polarized number of spins). In our case, the spin ensemble can be treated as a simple harmonic oscillator. ${ }^{7}$ The unique rigorous condition to reach the strong coupling regime ${ }^{8-11}$ is given by $g_{c} \gg \gamma_{s} \gg \kappa_{c}$, where $\kappa_{c}$ and $\gamma_{s}$ are the resonator and emitter damping rates, respectively. Another way to describe the strength of the coupling between the spins and the cavity mode is given by the cooperative factor, where the strong coupling is measured by $C=g_{c}{ }^{2} /\left(2 \kappa_{c} \gamma_{s}\right) \gg 1$. Frequency splitting or anti-crossing may be seen from the reflected or transmitted signal. It does

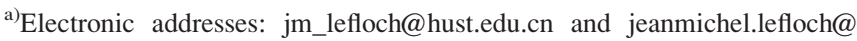
uwa.edu.au
}

not necessarily mean that $g_{c}>\gamma_{s}$, and $g_{c}<\kappa_{c}$ and thus that light-matter coherent information can be transferred. ${ }^{10}$ Relative to quantum-metrology, achieving the strong coupling regime with spin ensemble would give a higher sensitivity for detecting the signal absorbed or emitted by the spins. ${ }^{12}$ Recently, high-Q superconducting coplanar waveguide (CPW) resonators were used as read-out of flux qubit, ${ }^{13}$ transmons, ${ }^{14,15}$ or spin defects. ${ }^{16-19}$ The aim is to establish coherent transfer of information, by coupling two different quantum systems with a longer quantum coherence. The use of 3D superconducting cavities helps reaching longer coherence time but imposes many challenges in their realization as well as their future implementation. It has also been demonstrated that strong coupling to an ensemble of diamond nitrogen vacancy $\left(\mathrm{NV}^{-}\right)$spins is experimentally feasible. ${ }^{12,14,17,20,21}$

In this paper, we carefully determine the interaction between the electromagnetic field with an enhanced number of polarized spins ${ }^{7}$ and the resonator topology. We give a detailed numerical study of the $3 \mathrm{D}$ microwave cavity designs for optimizing the collective coupling between a spin ensemble and a microwave photon. To validate our design, we use $\mathrm{NV}^{-}$spin ensemble and compare against the previous 1D superconducting planar cavities. ${ }^{17,21}$ 


\section{PROOF OF PRINCIPLE ANALYSIS}

\section{A. Spin ensemble description}

Among all atomic-like solid state systems, we here focus on a well-known spin defect in diamond, the negatively charged state of the nitrogen vacancy center $\left(\mathrm{NV}^{-}\right) . \mathrm{NV}^{-}$has recently attracted a great interest, due to the unique properties of its ground state spin. The latter is an individually addressable solid state quantum bit at room temperature, ${ }^{13,19}$ with a very long coherence time. ${ }^{15}$ Also, quantum control using an optical read-out ${ }^{13,22}$ can be implemented. $\mathrm{NV}^{-}$center in diamond is constituted by a substitutional nitrogen atom close to a carbon vacancy having trapped an additional electron (Fig. 1 ); its electronic ground state $\operatorname{spin} S=1$, with the state $\mathrm{m}_{s}=0$ and $\mathrm{m}_{s}= \pm 1$, is separated by $2.87 \mathrm{GHz}$ at zero magnetic field. ${ }^{23}$ In the case of $\mathrm{NV}^{-}, m_{0}=g_{N V} \mu_{B}$ is the electron magnetic moment, where $\mu_{B}$ is the electron Bohr magneton, $g_{N V}=2.0028(\mathrm{~S}=1)$ is the NV Landé factor, owing to a ground state electron spin Hamiltonian given by $H_{N V}=m_{0} \mathbf{B} . \mathbf{S}+$ S.D.S. $\mathbf{B}$ is an external DC magnetic field and $\mathbf{D}$ is the zero field splitting for the axial component along $\mathrm{NV}$ axis $\left(\frac{D}{h}=2.877 \mathrm{GHz}\right)$. The hyperfine coupling to the ${ }^{14} \mathrm{~N}$ or ${ }^{13} \mathrm{C}$ nuclear spin is neglected for simplicity.

\section{B. Electromagnetic wave distribution in cavities}

\section{Cavity to spin interaction}

A microwave cavity consists of a metallic enclosure that confines electromagnetic fields in the microwave region of the spectrum. The structure is either unloaded or loaded with one or more dielectric materials. For single spin coupling, the coupling strength depends on the cavity mode volume, whereas, for ensemble of spins, the collective coupling depends only on the filling factor and the number of polarized spins. ${ }^{14}$ The coupling interaction between spins and the microwave field of the cavity can be described as a basic spin harmonic oscillator. The collective coupling strength can be expressed from the basic spin harmonic oscillator approximation, as given by ${ }^{14}$

$$
g_{c}=\frac{m_{0}}{2} \sqrt{\frac{\rho \mu_{0} \omega_{c} p_{m}}{\hbar}},
$$

where $\rho$ is the number of spins per unit volume, $\omega_{c}$ is the cavity resonance frequency, and $\mu_{0}$ is the vacuum permeability.

The cavity magnetic filling factor, $p_{m}$, describes the ACmagnetic field confinement in a particular volume of spins

$$
p_{m}=\frac{W_{m_{\text {material }}}}{W_{m_{\text {Total }}}}=\frac{\iiint_{V_{\text {material }}} \mu_{r} H \cdot H^{*} d v}{\iiint_{V_{\text {Total }}} \mu_{r}(v) H \cdot H^{*} d v},
$$

with $\mu_{r}$ being the relative permeability, which is in our case equals to $1 . p_{m}$ enters in increasing the collective spin coupling $g_{c}$, see Eq. (1), as the cavity electromagnetic mode must be highly confined within the spins volume density. We also assume that the spins volume density is uniform within the volume, thus it is not entering in the magnetic filling factor calculations.

\section{Cavity parameters determination}

We present here topologies that allow specifically the insertion of a bulk diamond sample rich in NV-spins, where the magnetic field confinement $\left(p_{m}\right)$ is the highest. The dielectrics loss (diamond and if applicable, the dielectric host) contributes to the resonator Q-factor. This dielectric loss is characterized by the microwave electric field confinement $\left(p_{e}\right)$ and its intrinsic loss mechanism $(\tan \delta)$

$$
p_{e}=\frac{W_{e_{\text {material }}}}{W_{e_{\text {Total }}}}=\frac{\oiiint \iint_{V_{\text {material }}} \epsilon_{\text {material }} E \cdot E^{*} d v}{\iiint_{V_{\text {Total }}} \epsilon(v) E \cdot E^{*} d v},
$$

where $\epsilon$ is the relative dielectric permittivity.

The dielectric loss dominates the cavity photons damping rate. Eventually, it drives the information encoded in the
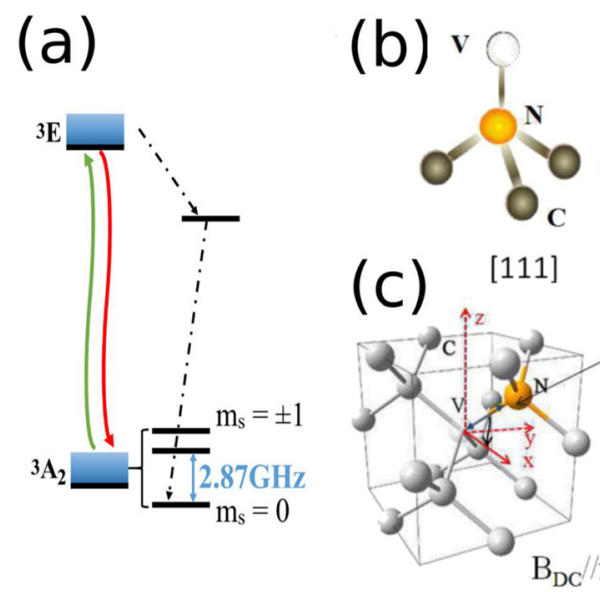
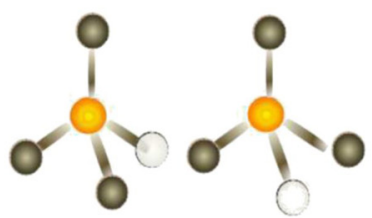

$[\overline{1} \overline{1} 1]$
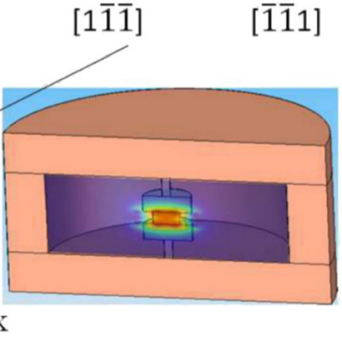

FIG. 1. (a)Simplified energy diagram of the NV center in diamond, showing the ground state spin level splitting and the excited state radiative and nonradiative transitions. (b) NV axis, four possible orientations, referred to the diamond crystallographic axis. (c) NV shown with its crystallographic location in a diamond cell (left) along one of the crystallographic directions $\langle 111\rangle$, while $\mathrm{z}$ indicated the main c-axis [001]. We consider that the bulk diamond $(3 \times 3$ $\times 1.5 \mathrm{~mm}$ ) is placed in the cavity (example shown of a double-split resonator) with the correct orientation to achieve only two sub-ensemble of NV defects aligned at $45^{\circ}$ with the DC applied magnetic $\mathrm{B}_{\mathrm{DC}}$ field. 
spin. The resonator damping rate $\kappa_{c}$ can be measured from the cavity resonance bandwidth

$$
Q_{0}=\frac{2 \pi \nu_{c}}{\kappa_{c}}=\frac{\omega_{c}}{\kappa_{c}},
$$

where $\nu_{c}$ is the resonance frequency, $\omega_{c}$ is the pulse cycle of the resonant cavity, and $Q_{0}$ is the unloaded Q-factor of the cavity, given by

$$
Q_{0}^{-1}=Q_{m e t}^{-1}+Q_{\text {diel } l_{\text {Dimond }}}^{-1}+Q_{\text {diel }}^{-1} h_{\text {host }},
$$

where $Q_{m e t}$ and $Q_{\text {diel }}$ correspond to the Q-factor of the metallic walls of the cavity and the Q-factor of the dielectrics (diamond and host) loaded into the cavity, respectively. ${ }^{24} \mathrm{We}$ also focus on reducing the limitations due to metallic losses estimated as

$$
Q_{m e t}^{-1}=\frac{R_{\text {surface }}}{G F},
$$

where $R_{\text {surface }}$ is related to the skin depth and metal conductivity of the cavity. And the geometric factor (GF) represents the microwave magnetic field distribution along the cavity surface.

Finally,

$$
Q_{\text {diel }}^{-1}=p_{e} \tan \delta,
$$

where $\tan \delta$ is the loss term of the dielectric material (diamond or host).

\section{Simulation description}

In our simulations, we assume that the contribution from the spin average orientation with respect to DC applied Bfield is the same in all studied cavities design. In fact, in irradiation produced $\mathrm{NV}$ centers in diamond, care must be taken to position the diamond to achieve two sub-ensemble of $\mathrm{NV}$ defects aligned at $45^{\circ}$ with the DC applied magnetic $\left(\mathrm{B}_{D C}\right)$ field (Fig. 1). This condition is design independent as it can always be achieved by being just a matter of mechanical fitting of the cavity-diamond inside the cryo-refrigerator and within the B-field homogeneity. Actually, the B-field homogeneity, using a conventional magnet, is at least $1 \mathrm{~cm}$ DSV (Diameter Sphere Volume), corresponding to a volume big enough to fully cover the diamond sample. The rotation of the cavity, to align a particular NV-center orientation with the DC-applied magnetic field, does not modify its electromagnetic properties. Therefore, we assume that the same cavity-diamond positioning is used for all the designed cavities to optimize the number of the coupled spin subset and all the studied cavities rely on the same probed spin densities. In the case of bulk diamond, where preferentially aligned NV spins are grown, all the spins in the material could be effectively coupled to the cavity modes. ${ }^{25}$

We only consider cavity modes with an AC-magnetic field propagating through a diamond sample. In this case, this is a fair assumption as the field occupies the volume of the whole sample. All simulations of the different structures presented here are conducted using the materials' properties at $4 \mathrm{~K}$.
The interest to be as low temperature as possible, in the $\mathrm{mK}$ regime, is to maximize the chance to detect strong coupling by lowering down the microwave noise and optimizing the cavity Q-factor. The aim, in this investigation, is to find the resonator with the smallest bandwidth and the highest AC-magnetic field confinement for enhancing the magneticdipole interaction of the defects. Thus, in the microwave regime, to match both conditions, it is necessary to use single crystal low-loss dielectrics at a low temperature. In addition, the sub-Kelvin temperature experiment ensures that the spins are not thermally polarized. We used a simulation software based on the finite element analysis, developed for the past 20 years at the XLIM institute and specifically optimized for microwave resonators.

The simulated resonance is $2.87 \mathrm{GHz} \pm 20 \mathrm{MHz}$, matching the microwave driving frequency of the NV center from $m_{s}=0$ to $m_{s}= \pm 1$. The magnetic $p_{m}$ (Eq. (2)) and electric $p_{e}$ (Eq. (3)) field confinements, relative to the cavity mode resonances into the NV-center defect in diamond, are calculated to determine the proportion of fields being confined in the diamond sample.

\section{Cavity based on waveguide design}

Here, we first focused on waveguide-based technologies, to confine the AC-magnetic field. We compare cavity topologies by the extend of the magnetic field confinement in the diamond sample. Different topologies of waveguides were used in various experiments and fields of research, such as gyrotron, ${ }^{26}$ wedge, ridge, and L-shape, including the use of septas. ${ }^{27}$ These were adapted into cavities by closing both extrema of the guide with a metallic plane.

All these are interesting for this experiment as magnetic field constraint, frequency, and shape are important parameters. They highly confine the electromagnetic field into their centers. Quarter-wavelength ridge long and half-wavelength L-shape waveguides use a high capacitance effect to reduce the resonance frequency. This capacitance is generated by a high-electric field between the large inserted metallic pieces (bar or L-shape). The capacitance enables separating significant areas where the electric and magnetic fields are confined.

The wedges and septas introduce perturbations of the electromagnetic field pattern propagating in the cavity. These perturbations allow a better field confinement into a particular area. This technique suffers from lowering the Q-factor due to large metallic losses. The aforementioned cavities are illustrated in Fig. 2. Such cavities are big at this resonance frequency, resulting in a very low AC-magnetic field confinement into the diamond, and thus, lowering down the sensitivity to detect strong coupling.

\section{High-confinement microwave cavities}

Compared to 1D cavities, a wider range of possible design and realization parameters are proposed to reach strong coupling towards the ultra-strong coupling regime. ${ }^{28}$ These enable further fundamental studies on cavity quantum electrodynamics due to their design flexibility and permit to tailor different spins systems. 
(a)

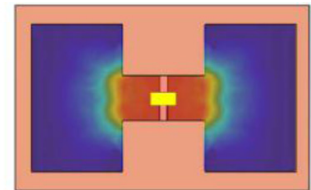

(c)

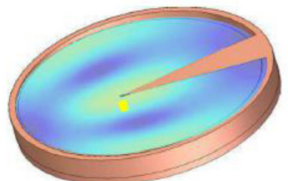

Low (b)

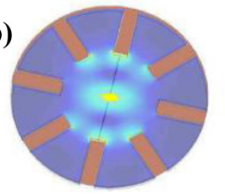

(d)

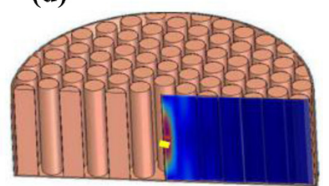

High

FIG. 2. Magnetic field density plots of the waveguide based technology, (a) ridge, (b) septa, (c) wedge, and (d) gyrotron. Due to scale ratio between the cavity and the diamond sample, the diamond (yellow square) is enhanced to illustrate the place where it is meant to be located.

\section{High- $Q$ dielectric resonators}

We previously designed the 3D cavities such as Transverse Electric (TE), ${ }^{29,30}$ lumped reentrant, ${ }^{27,31-34}$ Fabry-Perot (FP), ${ }^{35-38}$ and Whispering Gallery Mode $(\mathrm{WGM})^{39-41}$ resonators. High Q-factor cavities, with a very narrow linewidth, are ideal for the strong coupling detection. For this purpose, we also consider other cavities, such as high-Q dielectric Bragg, photonic bandgap resonators, and whispering gallery cylindrical and spherical resonators (see Fig. 3), which however provide a very low AC-magnetic field confinement (less than $10^{-3}$ ).

At such a low frequency, WGM, Bragg, and band gap resonators are limited by their sizes compared to diamond dimensions. This prevents us from fulfilling the strong coupling conditions. In addition, to fully confine a high magnetic field into the diamond sample with WGM, it is necessary to excite a large azimuthal mode number, which then reduces the maximum possible confinement per field maxima (less than 0.03).

\section{Cylindrical symmetry reentrant cavities}

Cylindrical reentrant cavities are unique 3D-structures, where their electric and magnetic fields are located in separate parts of the cavity. Reentrant cavities, illustrated in

(a)

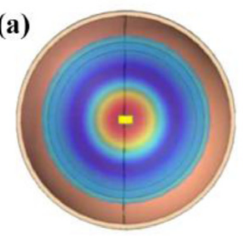

(e)

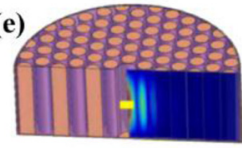

(b)

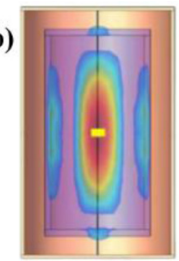

(f)

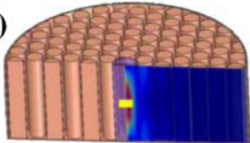

Low

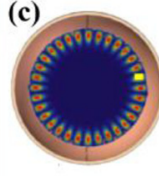

(d)
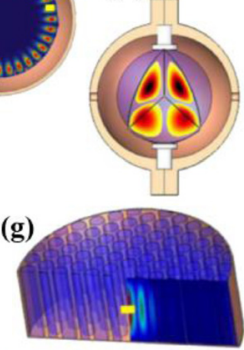

High

FIG. 3. Magnetic field density plots of (a), and (b) Bragg resonators, (c) and (d) whispering gallery modes both cylindrical and spherical symmetries, (e)-(g) photonic band gap resonators. ${ }^{42-46}$ For these different topologies, low loss dielectrics (purple color) have been used. ${ }^{4-49}$ Due to scale ratio between the cavity and the diamond sample sizes, the diamond (yellow square) is enhanced to illustrate the place where it is meant to be located.

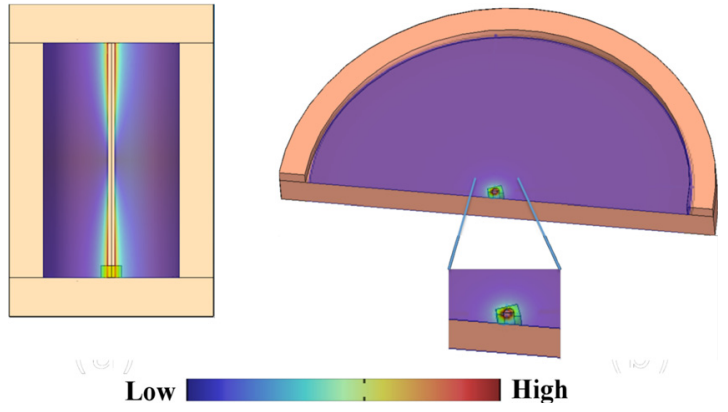

FIG. 4. Magnetic field density plots of (a) coaxial reentrant and (b) reentrant cavity. The diamond (yellow square) on figure (a) is enhanced to show the location. On figure (b) only the contour is shown to show the magnetic field.

Fig. 4, offer the advantage of a small size with a high confinement of magnetic field around the central post ${ }^{31-33}$ which imposes to drill a concentric hole into the sample to fit the cavity.

A double-post or periodic reentrant cavity can be built ${ }^{27,34}$ to place the sample in a high confined region of the cavity. With these topologies, the machining of the sample is not necessary but they exhibit low-Qs. As a consequence, we have studied other high-Q dielectric loaded cavities based on transverse electric (TE) modes.

\section{Double-split mode cavities}

When low-loss dielectric materials are inserted in transverse electric (TE) mode cavities, the cavity size can then be reduced and the AC-magnetic field confinement increases. ${ }^{30,50-52}$ One cavity design can respond to these criteria-the double-split cavity. It consists of a metallic enclosure loaded with two dielectric discs upside down, creating a gap in between for another dielectric material to be inserted (Figs. 5 and 6). Our proposed double-split cavity is composed of a diamond sample sandwiched between two discs of $\mathrm{TiO}_{2}$. The whole is inserted into a copper enclosure. The resonator mode is $\mathrm{TE}_{0,1, \delta}$. The large dielectric permittivity of $\mathrm{TiO}_{2}$ confines the field around the sample and allows a small size cavity for a $2.87 \mathrm{GHz}$ resonant frequency. The $\mathrm{TiO}_{2}$ low-microwave loss enables high-Qs, about 40 times higher than the reentrant cavity, with the same magnetic field confinement inside the sample (0.2).

For achieving strong coupling, we need to design a small volume cavity with a relatively high-Q and a very high AC-magnetic field confinement, where a dielectric host is not necessary to avoid uncertain coupling conditions.
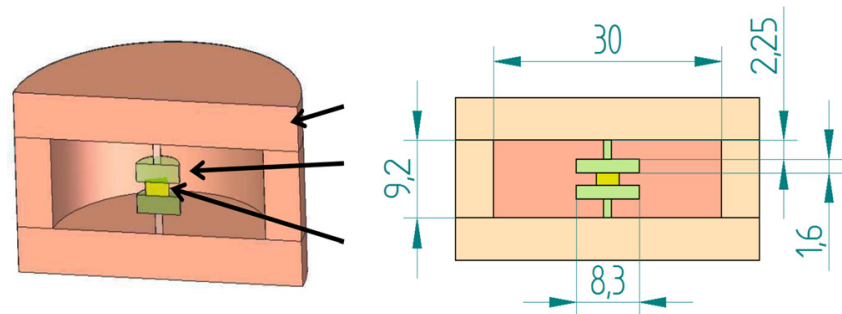

FIG. 5. (a) and (b) The double split-post resonator design, where $8 \mathrm{~mm}$ diameter c-axis parallel $\mathrm{TiO}_{2}$ discs confine the field into the diamond sample and hold tight in position the diamond sample. 


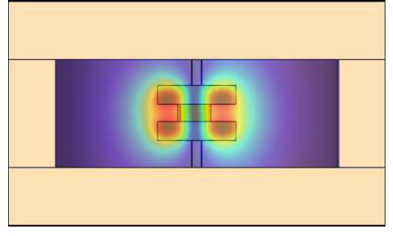

Low

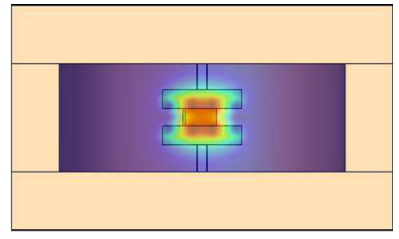

High
FIG. 6. (a) and (b) The density plots of the electric field and magnetic field, respectively. The color coding from blue to red corresponds to low to high intensity field.

\section{Development of a hybrid cavity}

The hybrid cavity design is a combination of topologies and aims at reducing cavity size and preventing the use of an additional dielectric, while maintaining a high AC-magnetic field confinement. For that particular purpose, the waveguide-based technology brings techniques to manipulate the field pattern and direction. The reentrant cavity does not require dielectric and is of small size. It also enables a high-field confinement. Combining the reentrant cavity with the waveguide, wedge, and septa topologies, ${ }^{53-55}$ we finally design an unloaded hybrid cavity (Figs. 7 and 8 ).

To reduce the size of the cavity, we use the electric symmetry properties of a transverse electric cavity mode through a pi-wedge design. Then the insertion of a pin (septa) through the height of the cavity modifies the wave propagation and creates a short-circuit. It automatically draws currents and intensifies the magnetic field. Finally, to reduce the frequency of the cavity down to $2.87 \mathrm{GHz}$, a large capacitive effect has to be created such as a reentrant cavity. The mushroom shape of the pin has no other function than reducing the metallic losses of the cavity. Due to the constructive combination of different technologies, a maximum magnetic field confinement into the diamond (0.47) can be achieved.

\section{VALIDATION OF MODEL AND ESTIMATION OF PERFORMANCE}

In this section, we provide the results for the most promising cavities loaded with the NV diamond sample: TE double split resonator and the hybrid cavity, shown in Figs. 5-8, respectively. The 1D CPW cavities ${ }^{21}$ are used as a reference to validate the calculations. The results of the simulations are summarized in Table I, where only the best results for the same mode are reported in comparison with the 1D CPW resonator. ${ }^{21}$ Two structures have clear interesting features for achieving strong coupling. First, the double split-post
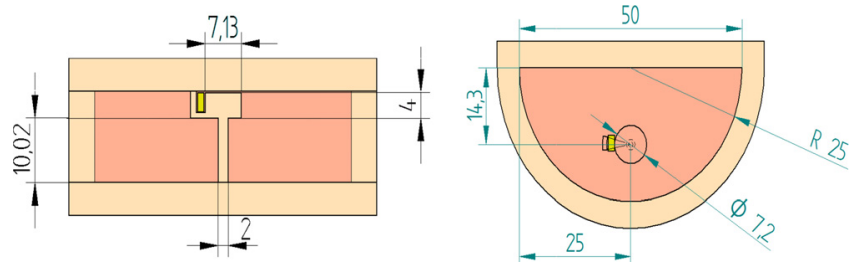

FIG. 7. (a) and (b) The hybrid cavity design with the inserted diamond sample.

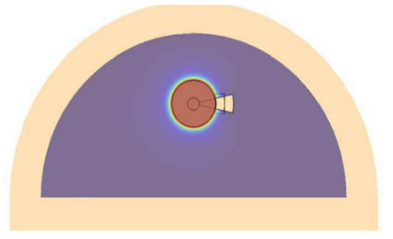

Low

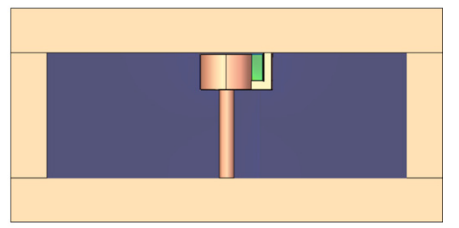

High
FIG. 8. (a) and (b) represent both the density plots of electric field (confined within the gap formed between the post and the top lid of the cavity) and the magnetic field (highly confined into the diamond sample). The color coding from blue to red corresponds to low to high intensity field.

resonator, shown in Figs. 5 and 6, has a very high-Q factor with a minimum of magnetic field confinement of 0.2 into the diamond sample. This design requires to have a large number of NVs. Second, the unloaded hybrid cavity, presented in Figs. 7 and 8, allows confining the magnetic field to 0.47 which is the highest field concentrated enabling structure. This means less NVs are necessary to achieve strong coupling. In such a structure, ultra-strong coupling could then be considered.

The linewidth of the spins compared with the cavity bandwidth has to be greater, as this cavity suffers from being low-Q. The cavity transmission mode showing frequency splitting once the cavity mode couples with the spin ensemble is given by

$$
\left|S_{11}\right|^{2}=\left|1+\frac{\kappa_{e}}{i\left(\omega-\omega_{c}\right)-\kappa+g_{c}^{2} /(i \Delta-\gamma)}\right|^{2},
$$

where $\kappa_{e}=\alpha \kappa_{c}$ is the external loss.

In Fig. 9, we illustrate the computed reflection spectra (from Eq. (8) and Table I) of an ensemble of NV-spins for the case of a coplanar resonator with small volume, corresponding to the reported measurements ${ }^{21}$ and for our hybrid cavity design with large volume. Even though the latter exhibits a lower Q-factor, the magnetic field confinement is higher, thus increasing the coupling strength significantly.

We assume the density of spins $\rho=1.2 \times 10^{6} \mu \mathrm{m}^{-3}$, which is typically achieved in high pressure high temperature (HPHT) diamond, after high energy electron/neutron irradiation or ion implantation. ${ }^{17,21}$ This value is usually measured by using confocal microscopy, and comparing the photo-luminescence (PL) of ensemble to a single defect. $\gamma_{s} / 2 \pi$ is the FWHM linewidth of the ESR lines of NV spins ensemble, proportional to the inverse of the spin dephasing time or phase relaxation time $\left(T_{2}^{*}\right)$. The ESR linewidth of the $\mathrm{NV}^{-}$center in diamond is sensitive to temperature and irradiation dose. For our simulations, we assume $\gamma_{s} / 2 \pi \approx 3 \mathrm{MHz}$ as we operate at low temperature. This is the expected broadening due to dipolar interactions with the neighboring ${ }^{14} \mathrm{~N}$ electronic spin $(\mathrm{S}=1 / 2)$. Typically in $100 \mathrm{ppm}$ HPHT diamond, we may expect $\gamma_{s} \approx 18.84 \mathrm{MHz}$.

A model with two-coupled oscillators describes the change in cavity frequency $\omega$ and cavity half-width once the cavity couples with spins in the presence of a DC magnetic field $B$ 
TABLE I. Summary of designed and computational parameters to determine the performance of each electromagnetic mode and cavities $\left(\mathrm{p}_{m}, \mathrm{p}_{e}, \mathrm{Q}_{0}\right)$ and also predict the coupling strength $g_{c}$ and the cooperative factor C. Mode denotes the electromagnetic mode structure the calculations are related to. We mostly used as a host for diamond, ${ }^{56} \mathrm{Al}_{2} \mathrm{O}_{3}$ (Refs. 47 and 57) (sapphire), $\mathrm{TiO}_{2}$ (Ref. 48) (rutile), and fused silica. ${ }^{58}$ We use copper ${ }^{59}$ for the cavity walls (Rs $=5.77 \mathrm{~m} \Omega$ ). All simulations are conducted at $4 \mathrm{~K}$, and the resonance frequency is $2.87 \mathrm{GHz} \pm 20 \mathrm{MHz}$.

\begin{tabular}{|c|c|c|c|c|c|c|}
\hline Mode & $p_{m} \times 10^{3}$ & $p_{e} \times 10^{3}$ & $Q_{0}$ & $g_{c}(\mathrm{MHz})$ & $\mathrm{V}\left(\mathrm{cm}^{3}\right)$ & $\mathrm{C}$ \\
\hline \multicolumn{7}{|c|}{ Double-split resonator-Figs. 5 and 6} \\
\hline $\mathrm{TE}^{48,50}$ & 84 & 0.63 & 127000 & 43 & 14 & 348 \\
\hline $\mathrm{TE}^{\mathrm{a})}($ Refs. 48 and 52) & 207 & 0.02 & 300000 & 68 & 106 & 2027 \\
\hline \multicolumn{7}{|c|}{ Reentrant cavities_-Fig. 4} \\
\hline $\mathrm{TM}^{33}$ & 100 & 0.35 & 1000 & 47 & 36 & 3.3 \\
\hline $\mathrm{TM}^{\mathrm{b})}$ (Refs. 31 and 33) & 230 & 0.26 & 1,250 & 71.5 & 36 & 9.4 \\
\hline $\mathrm{TM}^{34,60}$ & 270 & 0.27 & 500 & 77.5 & 36 & 4.4 \\
\hline \multicolumn{7}{|c|}{ Unloaded hybrid cavities (new topology)_Figs. 7 and 8} \\
\hline Copper & 471 & 49 & 500 & 102 & 14 & 7.7 \\
\hline With $\mathrm{Nb}$ post & 469 & 49 & 1000 & 102 & 14 & 15.3 \\
\hline \multicolumn{7}{|c|}{ Coplanar resonator-small volume } \\
\hline Ref. 21 & 119 & 215 & 1905 & 51 & 0.2 & 7.4 \\
\hline
\end{tabular}

${ }^{\text {a) }}$ Denotes the diamond-size is reduced to $1 \times 1 \times 0.25 \mathrm{~mm}$

${ }^{b)}$ Denotes the post of the cavity is made of Niobium $(\mathrm{Nb})$

$$
\omega=\omega_{c}+g_{c}^{2} \Delta /\left(\Delta^{2}+\gamma_{s}^{2}\right),
$$

where $\Delta=m_{o} \times\left(B-B_{r}\right) / \hbar$ is the field detuning from the resonant DC magnetic field $B_{r}$.

\section{DISCUSSION}

In summary, we have presented the 3D-cavities for achieving strong coupling at the exact transition of the NVdiamond ground state spin resonance; we verified the modes through the numerical finite-element modeling and validated our models comparing the obtained designed cavities parameters with some experimental realizations. ${ }^{17,30,34} \mathrm{We}$ also introduced a variety of topologies. We designed novel structures based on an unloaded hybrid cavity that confines four times the magnetic field into the diamond sample. It can provide twice the cooperative coupling, compared to small volume coplanar waveguides (CPW). This new type of cavity exhibits the highest magnetic field confinement in microwave cavities. In addition, this new cavity design can have potential applications in testing fundamental physics such as paraphoton detection. ${ }^{61}$ From our investigation, results show that strong coupling could also be achieved by a double-post cavity, where the collective coupling strength can be of the same order of the CPW but with 200 times better cooperative factor. The NV centers also offer access to an additional optical transition, which can be of interest in the $3 \mathrm{D}$ cavities with optical access to transfer quantum information from optical to microwave. The cavity designs allowing strong coupling and presented in this paper cannot provide a direct optical access, further investigation would be required.

The 3D-microwave cavities presented in this paper have a wide range of design parameters; they can also be applied to emerging similar qubits in silicon carbide ${ }^{62-64}$ or $\mathrm{Ce}^{3+}$ in YAG. ${ }^{65}$ The best cavities investigated here will certainly help by leading to even stronger couplings using other spins available in the solid state with much narrower linewidths than that of the NV centers, enabling new physics tests. If we were to use this particular design principle at different temperatures, the magnetic field confinement would remain identical within $5 \%$. Conversely, at different temperatures, the Q-factor of these cavities will strongly depend on the metallic enclosure conductivity or on its dielectric loss mechanism in the case of double-split resonators. Microwave temperature noise will increase with increasing temperatures, limiting the use of microwave cavities at room temperature.

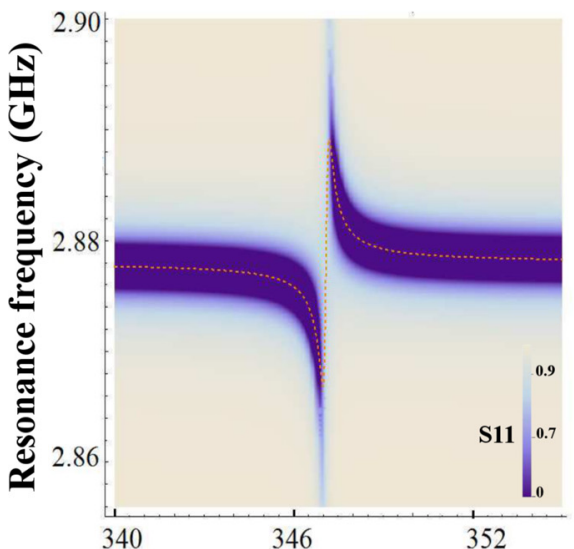

Applied magnetic field B (mT)

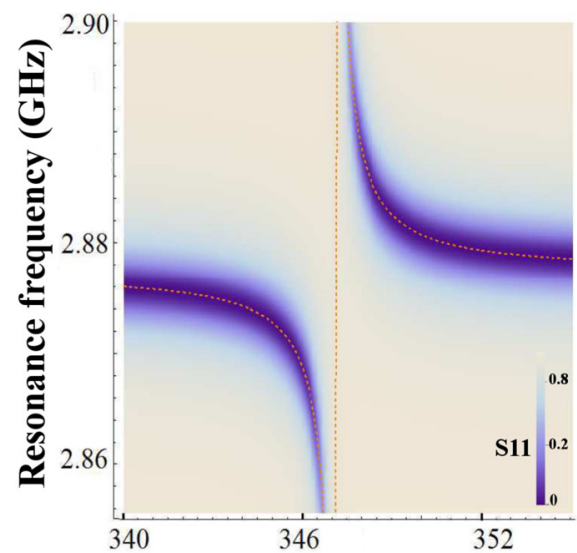

Applied magnetic field B (mT)
FIG. 9. $\left|S_{11}\right|^{2}$ spectrum for (a) CPW resonator, (b) hybrid cavity, our new topology with niobium post to reduce losses. The dashed lines correspond to Eq. (9). 


\section{ACKNOWLEDGMENTS}

This research was jointly supported under the Australian Research Council funding scheme: Laureate Fellowship (Project No. FL0992016), Centre of Excellence Engineered Quantum Systems (Project No. CE110001013), the French Research Agency (CNRS), and Labex Sigma-Lim (No. ANR-10-LABX-0074-01). The authors also thank le Conseil Régional du Limousin, cluster de calcul CALI (Calcul en Limousin) and the UWA Research Collaboration Award scheme.

${ }^{1}$ D. D. Awschalom, L. C. Bassett, A. S. Dzurak, E. L. Hu, and J. R. Petta, Science 339, 1174-1179 (2013).

${ }^{2}$ W. Pfaff, B. J. Hensen, H. Bernien, S. B. van Dam, M. S. Blok, T. H. Taminiau, M. J. Tiggelman, R. N. Schouten, M. Markham, D. J. Twitchen, and R. Hanson, Science 345, 532-535 (2014).

${ }^{3}$ J. Taylor, P. Cappellaro, L. Childress, L. Jiang, D. Budker, P. Hemmer, A. Yacoby, R. Walsworth, and M. Lukin, Nat. Phys. 4, 810-816 (2008).

${ }^{4}$ G. Kucsko, P. Maurer, N. Yao, M. Kubo, H. Noh, P. Lo, H. Park, and M. Lukin, Nature 500, 54-58 (2013).

${ }^{5}$ F. Brennecke, T. Donner, S. Ritter, T. Bourdel, M. Köhl, and T. Esslinger, Nature 450, 268-271 (2007).

${ }^{6}$ Y. Colombe, T. Steinmetz, G. Dubois, F. Linke, D. Hunger, and J. Reichel, Nature 450, 272-276 (2007).

${ }^{7}$ A. Imamoğlu, Phys. Rev. Lett. 102, 083602 (2009).

${ }^{8}$ R. Schoelkopf and S. Girvin, Nature 451, 664-669 (2008).

${ }^{9}$ J. M. Fink, "Quantum nonlinearities in strong coupling circuit QED," $\mathrm{Ph} . \mathrm{D}$. thesis (University of Vienna, 2010).

${ }^{10}$ E. Abe, H. Wu, A. Ardavan, and J. J. Morton, Appl. Phys. Lett. 98, 251108 (2011).

${ }^{11}$ G. Boero, G. Gualco, R. Lisowski, J. Anders, D. Suter, and J. Brugger, J. Magn. Reson. 231, 133-140 (2013).

${ }^{12}$ Y. Kubo, I. Diniz, C. Grezes, T. Umeda, J. Isoya, H. Sumiya, T. Yamamoto, H. Abe, S. Onoda, T. Ohshima et al., Phys. Rev. B 86, 064514 (2012).

${ }^{13}$ P. Neumann, R. Kolesov, B. Naydenov, J. Beck, F. Rempp, M. Steiner, V. Jacques, G. Balasubramanian, M. Markham, D. Twitchen et al., Nat. Phys. 6, 249-253 (2010).

${ }^{14}$ D. Schuster, A. Sears, E. Ginossar, L. DiCarlo, L. Frunzio, J. Morton, H. Wu, G. Briggs, B. Buckley, D. Awschalom et al., Phys. Rev. Lett. 105, 140501 (2010).

${ }^{15}$ G. Balasubramanian, P. Neumann, D. Twitchen, M. Markham, R. Kolesov, N. Mizuochi, J. Isoya, J. Achard, J. Beck, J. Tissler et al., Nat. Mater. 8, 383-387 (2009).

${ }^{16}$ J. Healey, T. Lindström, M. Colclough, C. Muirhead, and A. Y. Tzalenchuk, Appl. Phys. Lett. 93, 043513 (2008).

${ }^{17}$ X. Zhu, S. Saito, A. Kemp, K. Kakuyanagi, S.-i. Karimoto, H. Nakano, W. J. Munro, Y. Tokura, M. S. Everitt, K. Nemoto et al., Nature 478, 221-224 (2011).

${ }^{18}$ H. Paik, D. I. Schuster, L. S. Bishop, G. Kirchmair, G. Catelani, A. P. Sears, B. R. Johnson, M. J. Reagor, L. Frunzio, L. I. Glazman, S. M. Girvin, M. H. Devoret, and R. J. Schoelkopf, Phys. Rev. Lett. 107, 240501 (2011).

${ }^{19}$ N. Bar-Gill, L. M. Pham, A. Jarmola, D. Budker, and R. L. Walsworth, Nat. Commun. 4, 1743 (2013).

${ }^{20}$ R. Amsüss, C. Koller, T. Nöbauer, S. Putz, S. Rotter, K. Sandner, S. Schneider, M. Schramböck, G. Steinhauser, H. Ritsch et al., Phys. Rev. Lett. 107, 060502 (2011).

${ }^{21}$ Y. Kubo, F. Ong, P. Bertet, D. Vion, V. Jacques, D. Zheng, A. Dréau, J.-F. Roch, A. Auffèves, F. Jelezko et al., Phys. Rev. Lett. 105, 140502 (2010).

${ }^{22}$ L. Childress, M. G. Dutt, J. Taylor, A. Zibrov, F. Jelezko, J. Wrachtrup, P. Hemmer, and M. Lukin, Science 314, 281-285 (2006).

${ }^{23}$ M. Doherty, F. Dolde, H. Fedder, F. Jelezko, J. Wrachtrup, N. Manson, and L. Hollenberg, Phys. Rev. B 85, 205203 (2012).

${ }^{24}$ J.-M. Le Floch, Y. Fan, G. Humbert, Q. Shan, D. Férachou, R. BaraMaillet, M. Aubourg, J. G. Hartnett, V. Madrangeas, D. Cros et al., Rev. Sci. Instrum. 85, 031301 (2014).

${ }^{25}$ J. Michl, T. Teraji, S. Zaiser, I. Jakobi, G. Waldherr, F. Dolde, P. Neumann, M. W. Doherty, N. B. Manson, J. Isoya, and J. Wrachtrup, Appl. Phys. Lett. 104, 102407 (2014).
${ }^{26}$ Y. Zhang, S. Yu, L. Zhang, T. Zhang, Y. Yang, and H. Li, IEEE Trans. Plasma Sci. 43, 1018-1023 (2015).

${ }^{27}$ V. E. Boria and B. Gimeno, IEEE Microwave Mag. 8, 60-70 (2007).

${ }^{28}$ A. Moroz, Ann. Phys. 340, 252-266 (2014).

${ }^{29}$ J.-M. Le Floch, C. Bradac, N. Nand, S. Castelletto, M. E. Tobar, and T. Volz, Appl. Phys. Lett. 105, 133101 (2014).

${ }^{30}$ S. Probst, A. Tkalčec, H. Rotzinger, D. Rieger, J.-M. Le Floch, M. Goryachev, M. E. Tobar, A. V. Ustinov, and P. A. Bushev, Phys. Rev. B 90, 100404 (2014)

${ }^{31}$ W. W. Hansen, J. Appl. Phys. 9, 654-663 (1938).

${ }^{32}$ S. K. Remillard, A. Hardaway, B. Mork, J. Gilliland, and J. Gibbs, Prog. Electromagn. Res. B 15, 175-195 (2009).

${ }^{33}$ J.-M. Le Floch, Y. Fan, M. Aubourg, D. Cros, N. Carvalho, Q. Shan, J. Bourhill, E. Ivanov, G. Humbert, V. Madrangeas et al., Rev. Sci. Instrum. 84, 125114 (2013)

${ }^{34}$ D. L. Creedon, J.-M. Le Floch, M. Goryachev, W. G. Farr, S. Castelletto, and M. E. Tobar, Phys. Rev. B 91, 140408 (2015).

${ }^{35}$ C. A. Flory and R. C. Taber, IEEE Trans. Ultrason., Ferroelectr. Freq. Control 44, 486-495 (1997).

${ }^{36}$ J.-M. Le Floch, M. E. Tobar, D. Cros, and J. Krupka, IEEE Trans. Ultrason., Ferroelectr. Freq. Control 54, 2689-2695 (2007).

${ }^{37}$ J.-M. le Floch, M. E. Tobar, D. Mouneyrac, D. Cros, and J. Krupka, Appl. Phys. Lett. 91, 142907 (2007).

${ }^{38}$ M. E. Tobar, J.-M. Le Floch, D. Cros, J. Krupka, J. D. Anstie, and J. G. Hartnett, IEEE Trans. Ultrason., Ferroelectr. Freq. Control 51, 1054-1059 (2004).

${ }^{39}$ E. Snitzer, JOSA 51, 491-498 (1961).

${ }^{40} \mathrm{O}$. Di Monaco, Y. Kersalé, and V. Giordano, IEEE Microwave Guided Wave Lett. 10, 368-370 (2000).

${ }^{41}$ J.-M. Le Floch, J. D. Anstie, M. E. Tobar, J. G. Hartnett, P.-Y. Bourgeois, and D. Cros, Phys. Lett. A 359, 1-7 (2006).

${ }^{42}$ J. Sirigiri, K. Kreischer, J. Machuzak, I. Mastovsky, M. Shapiro, and R. Temkin, Phys. Rev. Lett. 86, 5628-5631 (2001).

${ }^{43}$ H. Altug and J. Vučković, Appl. Phys. Lett. 84, 161-163 (2004).

${ }^{44}$ G. Humbert, J.-M. Le Floch, D. Mouneyrac, D. Férachou, M. Aubourg, M. Tobar, D. Cros, and J.-M. Blondy, Appl. Phys. Lett. 96, 051108 (2010).

${ }^{45}$ D. Férachou, G. Humbert, J.-M. Le Floch, M. Aubourg, J.-L. Auguste, M. Tobar, D. Cros, and J.-M. Blondy, Electron. Lett. 47, 805-807 (2011).

${ }^{46}$ M. N. Armenise, C. E. Campanella, C. Ciminelli, F. Dell'Olio, and V. M. N. Passaro, Physics Procedia 3(1), 357-364 (2010).

${ }^{47}$ J. Krupka, K. Derzakowski, M. Tobar, J. Hartnett, and R. G. Geyer, Meas. Sci. Technol. 10, 387 (1999).

${ }^{48}$ A. Luiten, M. Tobar, J. Krupka, R. Woode, E. Ivanov, and A. Mann, J. Phys. D: Appl. Phys. 31, 1383 (1998).

${ }^{49}$ J.-M. Le Floch, M. E. Tobar, D. Cros, and J. Krupka, Appl. Phys. Lett. 92, 032901 (2008).

${ }^{50}$ M. D. Janezic and J. Baker-Jarvis, IEEE Trans. Microwave Theory Tech. 47, 2014-2020 (1999)

${ }^{51}$ Q. Simon, V. Bouquet, W. Peng, J.-M. Le Floch, F. Houdonougbo, S. Députier, S. Weber, A. Dauscher, V. Madrangeas, D. Cros, and M. Guilloux-Viry, Thin Solid Films 517, 5940-5942 (2009).

${ }^{52}$ J.-M. Le Floch, F. Houndonougbo, V. Madrangeas, D. Cros, M. Guilloux-Viry, and W. Peng, J. Electromagn. Waves Appl. 23, 549-559 (2009).

${ }^{53}$ R. Chisholm, IRE Trans. Antennas and Propag. 7, 279-283 (1959).

${ }^{54}$ D. Budimir and C. W. Turner, Microwave Opt. Technol. Lett. 23, 311-312 (1999).

${ }^{55}$ N. N. Esfahani, P. Rezaee, K. Schünemann, R. Knöchel, and M. Tayarani, in 2011 International Conference on Electromagnetics in Advanced Applications (ICEAA) (IEEE, 2011), pp. 1086-1089.

${ }^{56}$ J.-M. Le Floch, R. Bara, J. G. Hartnett, M. E. Tobar, D. Mouneyrac, D. Passerieux, D. Cros, J. Krupka, P. Goy, and S. Caroopen, J. Appl. Phys. 109, 094103 (2011)

${ }^{57}$ D. L. Creedon, M. E. Tobar, J.-M. Le Floch, Y. Reshitnyk, and T. Duty, Phys. Rev. B 82, 104305 (2010).

${ }^{58}$ J. D. Anstie, J. G. Hartnett, M. E. Tobar, J. Winterflood, D. Cros, and J. Krupka, Meas. Sci. Technol. 14, 286 (2003).

${ }^{59}$ J. Molla, A. Ibarra, J. Margineda, J. Zamarro, and A. Hernandez, IEEE Trans. Instrum. Meas. 42, 817-821 (1993).

${ }^{60}$ M. Goryachev, W. G. Farr, D. L. Creedon, Y. Fan, M. Kostylev, and M. E. Tobar, Phys. Rev. Appl. 2, 054002 (2014).

${ }^{61}$ S. R. Parker, J. G. Hartnett, R. G. Povey, and M. E. Tobar, Phys. Rev. D 88, 112004 (2013). 
${ }^{62}$ W. F. Koehl, B. B. Buckley, F. J. Heremans, G. Calusine, and D. D. Awschalom, Nature 479, 84-87 (2011).

${ }^{63}$ H. Kraus, V. Soltamov, D. Riedel, S. Väth, F. Fuchs, A. Sperlich, P. Baranov, V. Dyakonov, and G. Astakhov, Nat. Phys. 10, 157-162 (2014).
${ }^{64}$ S. Castelletto, B. Johnson, V. Ivády, N. Stavrias, T. Umeda, A. Gali, and T. Ohshima, Nat. Mater. 13, 151-156 (2014).

${ }^{65}$ P. Siyushev, K. Xia, R. Reuter, M. Jamali, N. Zhao, N. Yang, C. Duan, N. Kukharchyk, A. Wieck, R. Kolesov et al., Nat. Commun. 5, 3895 (2014). 